\title{
Sets of exact 'logarithmic' order in the theory of Diophantine approximation*
}

\author{
Victor Beresnevich
}

MinsK

\author{
Detta Dickinson \\ YORK
}

Sanju Velani ${ }^{\dagger}$

LONDON

\begin{abstract}
For each real number $\alpha$, let $E(\alpha)$ denote the set of real numbers with exact order $\alpha$. A theorem of Güting states that for $\alpha \geq 2$ the Hausdorff dimension of $E(\alpha)$ is equal to $2 / \alpha$. In this note we introduce the notion of exact $t$-logarithmic order which refines the usual definition of exact order. Our main result for the associated refined sets generalizes Güting's result to linear forms and moreover determines the Hausdorff measure at the critical exponent. In fact, the sets are shown to satisfy delicate zero-infinity laws with respect to Lebesgue and Hausdorff measures. These laws are reminiscent of those satisfied by the classical set of well approximable real numbers, for example as demonstrated by Khintchine's theorem.
\end{abstract}

\section{$\S 1$ Background and statement of results.}

\section{$\S 1.1$ The classical theory.}

For each real number $\tau$, let $W(\tau)$ denote the set of real numbers which are $\tau$-well approximable, that is

$$
W(\tau):=\left\{x \in \mathbb{R}:|x-p / q| \leq|q|^{-\tau} \text { for infinitely many rationals } p / q\right\} .
$$

For a real number $x$, its exact order $\tau(x)$ is defined as follows:

$$
\tau(x):=\sup \{\tau: x \in W(\tau)\}
$$

It follows from Dirichlet's theorem in the theory of Diophantine approximation that $\tau(x) \geq 2$ for all $x \in \mathbb{R}$. For $\alpha \geq 2$, let $E(\alpha)$ denote the set of numbers with exact order $\alpha$; that is

$$
E(\alpha):=\{x \in \mathbb{R}: \tau(x)=\alpha\} .
$$

*Published: Math Annalen, 321 (2001) 253-273.

${ }^{\dagger}$ Royal Society University Research Fellow 
The set $E(\alpha)$ is equivalent to the set of real numbers $x$ for which Mahler's function $\theta_{1}(x)$ is equal to $\alpha-1$; the general function $\theta_{n}(x)$ is central to Mahler's classification of transcendental numbers (see [1,4]). In [4], Güting proved the following result:

Theorem (Güting). For $\alpha \geq 2, \operatorname{dim} E(\alpha)=2 / \alpha$.

Throughout, $\operatorname{dim} X$ will denote the Hausdorff dimension of the set $X$. For further details regarding Hausdorff dimension and measure see $\S 1.3$. At this point it is worth mentioning the classical result of Jarník and Besicovitch which states that $\operatorname{dim} W(\alpha)=2 / \alpha$. Thus, Güting's result says that the 'size' of the sets $E(\alpha)$ and $W(\alpha)$ expressed in terms of Hausdorff dimension are the same. In this note we give a short proof of Güting's theorem and show that the $s$-dimensional Hausdorff measure of the set $E(\alpha)$ at the critical exponent is infinity, that is

$$
\mathcal{H}^{\frac{2}{\alpha}}(E(\alpha))=\infty
$$

These exact order results are a simple consequence of our results on exact $t$ logarithmic order.

\section{$\S 1.2$ Linear forms and exact $t$-logarithmic order.}

Let $\psi: \mathbb{R}^{+} \rightarrow \mathbb{R}^{+}$be a real positive function. An $m \times n$ matrix $X=\left(x_{i j}\right) \in \mathbb{R}^{m n}$ is said to be $\psi$-well approximable if the system of inequalities

$$
\left|q_{1} x_{1 j}+q_{2} x_{2 j}+\ldots+q_{m} x_{m j}-p_{j}\right| \leq \psi(|\mathbf{q}|)|\mathbf{q}| \quad(1 \leq j \leq n)
$$

is satisfied for infinitely many vectors $\mathbf{q} \in \mathbb{Z}^{m}, \mathbf{p} \in \mathbb{Z}^{n}$. Here $|\mathbf{q}|$ denotes the supremum norm of the vector $\mathbf{q}$; i.e. $|\mathbf{q}|=\max \left\{\left|q_{1}\right|, \ldots,\left|q_{m}\right|\right\}$. The system

$$
q_{1} x_{1 j}+q_{2} x_{2 j}+\ldots+q_{m} x_{m j} \quad(1 \leq j \leq n)
$$

of $n$ real linear forms in $m$ variables $q_{1}, \ldots, q_{m}$ will be written more concisely as

$$
\mathbf{q} X
$$

where the matrix $X$ is regarded as a point in $\mathbb{R}^{m n}$. In view of this notation, the set of $\psi$-well approximable points will be denoted by

$$
W(m, n ; \psi):=\left\{X \in \mathbb{R}^{m n}:|\mathbf{q} X-\mathbf{p}| \leq \psi(|\mathbf{q}|)|\mathbf{q}| \text { for i.m. }(\mathbf{p}, \mathbf{q}) \in \mathbb{Z}^{n} \times \mathbb{Z}^{m}\right\},
$$

where 'i.m.' means 'infinitely many'. By definition, $|\mathbf{q} X-\mathbf{p}|=\max _{1 \leq j \leq n} \mid \mathbf{q} . X^{(j)}-$ $p_{j} \mid$ where $X^{(j)}$ is the j'th column vector of $X$. In the case when $\psi(r)=r^{-\tau}$ we denote $W(m, n ; \psi)$ by $W(m, n ; \tau)$ and note that when $m=n=1$ the set $W(1,1 ; \tau)$ corresponds to $W(\tau)$ in the one dimensional theory.

We now refine and extend the definition of exact order to linear forms. For $t \geq 1$, suppose $\alpha_{o}, \alpha_{1}, \ldots, \alpha_{t-1} \in \mathbb{R}$ are given. Then for $\tau \in \mathbb{R} \backslash\{0\}$ denote by $W_{t}(m, n ; \tau)$ the set of $X$ in $\mathbb{R}^{m n}$ for which the inequality

$$
|\mathbf{q} X-\mathbf{p}| \leq|\mathbf{q}|^{-\alpha_{o}+1} \prod_{i=1}^{t-1}\left(\log _{i}|\mathbf{q}|\right)^{-\alpha_{i}} \times\left(\log _{t}|\mathbf{q}|\right)^{-\tau}
$$


is satisfied for infinitely many $(\mathbf{p}, \mathbf{q}) \in \mathbb{Z}^{n} \times \mathbb{Z}^{m}$. Here and throughout $\log _{i} r$ denotes the iterated logarithm

$$
\underbrace{\log \ldots \log r}_{i \text { times }} r
$$

When $t=0$, we put

$$
W_{o}(m, n ; \tau):=W(m, n ; \tau) .
$$

For $X$ in $\mathbb{R}^{m n}$ and $t \geq 0$, let

$$
\tau_{t}(X):=\sup \left\{\tau: X \in W_{t}(m, n ; \tau)\right\}
$$

denote the exact $t$-logarithmic order of $X$. Furthermore, for $\alpha_{t} \neq 0$ let

$$
E_{t}\left(m, n ; \alpha_{t}\right):=\left\{X \in \mathbb{R}^{m n}: \tau_{t}(X)=\alpha_{t}\right\}
$$

denote the set of 'points' $X$ with exact $t$-logarithmic order $\alpha_{t}$. Here in the definition of $E_{t}\left(m, n ; \alpha_{t}\right)$ it is understood that on fixing $\alpha_{t}$ we have already fixed the real numbers $\alpha_{o}, \alpha_{1}, \ldots, \alpha_{t-1}$. Notice that in the case when $m=n=1$ and $t=0$, the set $E_{t}\left(m, n ; \alpha_{t}\right)$ is precisely the classical set of real numbers with exact order $\alpha_{o}$. In view of the analogue of Dirichlet's Theorem for linear forms, for any $X \in \mathbb{R}^{m n}$ there exist infinitely many $(\mathbf{p}, \mathbf{q}) \in \mathbb{Z}^{n} \times \mathbb{Z}^{m}$ such that

$$
|\mathbf{q} X-\mathbf{p}| \leq|\mathbf{q}|^{-\frac{m+n}{n}+1} .
$$

This implies that for any $\alpha_{o}<\frac{m+n}{n}$, the set $E_{t}\left(m, n ; \alpha_{t}\right)$ is empty. Thus without loss of generality we will always assume that

$$
\alpha_{o} \geq \frac{m+n}{n} .
$$

Suppose for the moment that $t \geq 1$ and $X \in E_{t}\left(m, n ; \alpha_{t}\right)$. It follows, by definition, that for any $\epsilon>0$

$$
|\mathbf{q} X-\mathbf{p}| \leq|\mathbf{q}|^{-\alpha_{o}+1} \prod_{i=1}^{t}\left(\log _{i}|\mathbf{q}|\right)^{-\alpha_{i}} \times\left(\log _{t}|\mathbf{q}|\right)^{\epsilon}
$$

for infinitely many $(\mathbf{p}, \mathbf{q}) \in \mathbb{Z}^{n} \times \mathbb{Z}^{m}$, and that

$$
|\mathbf{q} X-\mathbf{p}| \geq|\mathbf{q}|^{-\alpha_{o}+1} \prod_{i=1}^{t}\left(\log _{i}|\mathbf{q}|\right)^{-\alpha_{i}} \times\left(\log _{t}|\mathbf{q}|\right)^{-\epsilon}
$$

for all $(\mathbf{p}, \mathbf{q}) \in \mathbb{Z}^{n} \times \mathbb{Z}^{m}$ with $|\mathbf{q}|$ sufficiently large. Thus, given $\alpha_{o}, \alpha_{1}, \ldots, \alpha_{t}$ with $\alpha_{t} \geq 0$ we have the inclusion

$$
E_{t}\left(m, n ; \alpha_{t}\right) \subseteq E_{t-1}\left(m, n ; \alpha_{t-1}\right) .
$$

Thus as $t$ increases, the 'order' of approximation of points in $E_{t}\left(m, n ; \alpha_{t}\right)$ becomes more and more precise. The aim of this note is to completely describe the metric structure of the sets $E_{t}\left(m, n ; \alpha_{t}\right)$. In terms of dimension, we will see that the 'size' 
of $E_{t}\left(m, n ; \alpha_{t}\right)$ is only dependent on $\alpha_{o}$ regardless of the precision of approximation induced by the presence of $\log$ terms. However, the log terms do influence the Hausdorff measures.

\section{$\S 1.3$ The metric structure of the sets $E_{t}\left(m, n ; \alpha_{t}\right)$.}

The appropriate notion to 'best' describe the structure of the sets $E_{t}\left(m, n ; \alpha_{t}\right)$ is that of generalized Hausdorff measures. By this we mean, Hausdorff measures with respect to various dimension functions. A dimension function $f: \mathbb{R}^{+} \rightarrow \mathbb{R}^{+}$is an increasing, continuous function such that $f(r) \rightarrow 0$ as $r \rightarrow 0$. The Hausdorff measure with respect to the dimension function $f$ will be denoted throughout by $\mathcal{H}^{f}$ and is defined as follows. Suppose $F$ is a non-empty subset of $k$-dimensional Euclidean space $\mathbb{R}^{k}$. For $\rho>0$, a countable collection $\left\{C_{i}\right\}$ of Euclidean cubes in $\mathbb{R}^{k}$ with sidelength $L\left(C_{i}\right) \leq \rho$ for each $i$ such that $F \subset \bigcup_{i} C_{i}$ is called a $\rho$-cover for $F$. For a dimension function $f$ define $\mathcal{H}_{\rho}^{f}(F)=\inf \left\{\sum_{i} f\left(L\left(C_{i}\right)\right):\left\{C_{i}\right\}\right.$ is a $\rho$-cover of $\left.F\right\}$, where the infimum is over all $\rho$-covers. The Hausdorff measure $\mathcal{H}^{f}(F)$ of $F$ with respect to the dimension function $f$ is defined by

$$
\mathcal{H}^{f}(F):=\lim _{\rho \rightarrow 0} \mathcal{H}_{\rho}^{f}(F)=\sup _{\rho>0} \mathcal{H}_{\rho}^{f}(F)
$$

A simple consequence of the definition of $\mathcal{H}^{f}$ is the following useful fact:

Fact. If $f$ and $g$ are two dimension functions such that $f(r) / g(r) \rightarrow 0$ as $r \rightarrow 0$, then $\mathcal{H}^{f}(F)=0$ whenever $\mathcal{H}^{g}(F)<\infty$.

In the case that $f(r)=r^{s}(s \geq 0)$, the measure $\mathcal{H}^{f}$ is the usual $s$-dimensional Hausdorff measure $\mathcal{H}^{s}$ and the Hausdorff dimension $\operatorname{dim} F$ of a set $F$ is defined by

$$
\operatorname{dim} F:=\inf \left\{s: \mathcal{H}^{s}(F)=0\right\}=\sup \left\{s: \mathcal{H}^{s}(F)=\infty\right\}
$$

In particular when $s$ is an integer $\mathcal{H}^{s}$ is comparable to $s$-dimensional Lebesgue measure. For further details see $[2,3]$.

Returning to the problem of describing the metric structure of $E_{t}\left(m, n ; \alpha_{t}\right)$, the following function will play a crucial role. For any $\delta \geq 0$, we consider the dimension function $f_{\delta}$ given by

$$
f_{\delta}(r):=r^{d+\delta} \quad \text { if } \quad t=0
$$

where

$$
d:=(m-1) n+\frac{m+n}{\alpha_{o}}
$$

and

$$
f_{\delta}(r):=r^{d} \prod_{i=1}^{t-1}\left(\log _{i} r^{-1}\right)^{u\left(\alpha_{i}\right)} \times\left(\log _{t} r^{-1}\right)^{u\left(\alpha_{t}\right)-\delta} \quad \text { if } \quad t \geq 1,
$$

where for $1 \leq i \leq t$

$$
u\left(\alpha_{i}\right):=\frac{\alpha_{i}(m+n)}{\alpha_{o}}-1 .
$$


As mentioned in the previous section we assume without loss of generality that $\alpha_{o} \geq(m+n) / n$. For the case $\alpha_{o}$ strictly greater than $(m+n) / n$ we are able to give a complete metric description of the sets $E_{t}\left(m, n ; \alpha_{t}\right)$ :

Theorem 1 For $\alpha_{o}>\frac{m+n}{n}, \operatorname{dim} E_{t}\left(m, n ; \alpha_{t}\right)=d$. Furthermore

$$
\mathcal{H}^{f_{\delta}}\left(E_{t}\left(m, n ; \alpha_{t}\right)\right)=\left\{\begin{array}{ccc}
\infty & \text { if } & \delta=0 \\
0 & \text { if } \quad \delta>0
\end{array} .\right.
$$

Recall that the dimension functions $f_{\delta}$ depend on the values of $\alpha_{o}, \ldots, \alpha_{t}$. Thus the measure part of the theorem shows that the Hausdorff measure with respect to these functions gives a precise and natural tool for distinguishing between the sets $E_{t}\left(m, n ; \alpha_{t}\right)$ even though for a fixed $\alpha_{o}$ they have the same Hausdorff dimension. Also notice that $d<m n$, so the $m n$-dimensional Lebesgue measure of $E_{t}\left(m, n ; \alpha_{t}\right)$ is always zero.

In the case when $\alpha_{o}=(m+n) / n$, our approach yields partial results for the dimension and measure of $E_{t}\left(m, n ; \alpha_{t}\right)$. Recall that $m n$-dimensional Hausdorff measure $\mathcal{H}^{m n}$ is comparable to $m n$-dimensional Lebesgue measure.

Theorem 2 Suppose $\alpha_{o}=\frac{m+n}{n}$. If $t=0$ or $\alpha_{i}=1 / n$ for all $1 \leq i \leq t$, then $\operatorname{dim} E_{t}\left(m, n ; \alpha_{t}\right)=m n$ and

- $\mathcal{H}^{m n}\left(E_{t}\left(m, n ; \alpha_{t}\right)\right)=\infty$

- $\quad \mathcal{H}^{h}\left(E_{t}\left(m, n ; \alpha_{t}\right)\right)=0 \quad$ if $\quad r^{-m n} h(r) \rightarrow 0$ as $r \rightarrow 0$.

In all other cases $\mathcal{H}^{m n}\left(E_{t}\left(m, n ; \alpha_{t}\right)\right)=0$. In particular, if

$$
\alpha_{1}=\ldots=\alpha_{k-1}=1 / n \text { and } \quad \alpha_{k}>1 / n
$$

for some $1 \leq k \leq t$; then $\operatorname{dim} E_{t}\left(m, n ; \alpha_{t}\right)=m n$ and

$$
\mathcal{H}^{f_{\delta}}\left(E_{t}\left(m, n ; \alpha_{t}\right)\right)=\left\{\begin{array}{ccc}
\infty & \text { if } & \delta=0 \\
0 & \text { if } & \delta>0
\end{array} .\right.
$$

In the classical 'exact order' situation $(t=0)$, Theorems 1 and 2 together give a complete metric description of the set $E_{o}\left(m, n ; \alpha_{o}\right)$.

For $t \geq 1$, Theorem 2 is to some extent unsatisfactory. For example, when $\alpha_{1}<$ $1 / n$ the result gives no information regarding the dimension of $E_{t}\left(m, n ; \alpha_{t}\right)$ - all we know is that $E_{t}\left(m, n ; \alpha_{t}\right)$ is of zero $m n$-dimensional Lebesgue measure. However, if $\alpha_{1}>1 / n$ the result provides a complete metric description of $E_{t}\left(m, n ; \alpha_{t}\right)$. 


\section{$\S 1.4$ A general formalism.}

The Diophantine approximation properties of points $X$ in $E_{t}\left(m, n ; \alpha_{t}\right)$ are sandwiched between two approximating functions which are a product of iterated logarithms differing at the $t$-iterated logarithm. This is clearly a 'natural' refinement of the standard exact order situation. However, a more general, and in some sense a more natural approach is to characterize the approximation properties of points $X$ in $\mathbb{R}^{m n}$ with respect to two arbitrary approximating functions - clearly not all functions can be written as a product of iterated logarithms. More precisely, given two real, positive decreasing functions $\varphi$ and $\psi$ with $\varphi$ in some sense 'smaller' than $\psi$, consider the set $D(m, n ; \psi, \varphi)$ of points $X$ in $\mathbb{R}^{m n}$ for which

$$
|\mathbf{q} X-\mathbf{p}| \leq|\mathbf{q}| \psi(|\mathbf{q}|) \quad \text { infinitely often } ;
$$

and that

$$
|\mathbf{q} X-\mathbf{p}| \geq|\mathbf{q}| \varphi(|\mathbf{q}|) \quad \text { for all }|\mathbf{q}| \text { sufficiently large }
$$

In short,

$$
D(m, n ; \psi, \varphi):=W(m, n ; \psi) \backslash W(m, n ; \varphi) .
$$

In such generality one can not expect to describe the metric structure of $D(m, n ; \psi, \varphi)$ as completely as in the exact $t$-logarithmic situation where the functions $\varphi$ and $\psi$ are explicit. However, under reasonably mild conditions on the growth of the function $\psi$ and the degree to which $\varphi$ is smaller than $\psi$ we are able to completely describe the metric structure of $D(m, n ; \psi, \varphi)$. The general philosophy is that the more explicit the functions the finer the metric description of $D(m, n ; \psi, \varphi)$ one expects to obtain.

The notion of 'order' of a real, positive, decreasing function $\psi: \mathbb{R}^{+} \rightarrow \mathbb{R}^{+}$will be significant to our general framework. The order $\lambda(\psi)$ is defined as follows:

$$
\lambda(\psi):=\lim _{r \rightarrow \infty} \frac{-\log \psi(r)}{\log r} .
$$

In the exact $t$-logarithmic order situation, the approximating functions are of the form $\psi(r)=r^{-\alpha_{o}} \prod_{i=1}^{t}\left(\log _{i} r\right)^{-\alpha_{i}}$. Clearly in this case the order exists and $\lambda(\psi)=\alpha_{o}$. To simplify matters we will only state and prove an analogue of Theorem 1 in which we assumed $\alpha_{o}>(m+n) / n$. Recall that this assumption ensured that Hausdorff dimension of the sets under consideration is strictly less than $m n$ (the dimension of the ambient space) and therefore of $m n$-dimensional Lebesgue measure zero. Here we impose the condition that $\lambda(\psi)>(m+n) / n$.

Theorem 3 Let $\psi: \mathbb{R}^{+} \rightarrow \mathbb{R}^{+}$be a strictly decreasing and continuous function such that $\lambda(\psi)>(m+n) / n$. Let $\varphi: \mathbb{R}^{+} \rightarrow \mathbb{R}^{+}$be a decreasing function such that

$$
\varphi(r) \leq \psi\left(r \log _{k} r\right)
$$

for some integer $k \geq 1$ and $r$ sufficiently large. If $r^{m+n} \psi(r)^{n} \prod_{i=1}^{k-1} \log _{i} r$ is nonincreasing then

$$
\mathcal{H}^{f_{\delta}}(D(m, n ; \psi, \varphi))=\left\{\begin{array}{cc}
\infty & \text { if } \delta=0 \\
0 & \text { if } \delta>0
\end{array},\right.
$$


where $f_{\delta}$ is the dimension function given by $f_{\delta}(r):=r^{(m-1) n}\left(\psi^{-1}(r)\right)^{-m-n-\delta}$ if $k=1$ and if $k \geq 2$, then

$$
f_{\delta}(r):=r^{(m-1) n}\left(\psi^{-1}(r)\right)^{-m-n} \prod_{i=1}^{k-2}\left(\log _{i} \psi^{-1}(r)\right)^{-1} \times\left(\log _{k-1} \psi^{-1}(r)\right)^{-1-\delta} .
$$

Furthermore

$$
\operatorname{dim} D(m, n ; \psi, \varphi)=n(m-1)+(m+n) / \lambda(\psi)
$$

Remark. For the zero-infinity law, which is the main content of the theorem and the upper bound for the dimension we only require that $\lim \inf _{r \rightarrow \infty}-\log \psi(r) / \log r>$ $(m+n) / n$; i.e. a condition on the lower order of $\psi$. Also, any dimension function which is comparable to the explicit function $f_{\delta}$ stated in the theorem will have the desired properties. In fact, if $\lambda(\psi)<\infty$ then for $r$ sufficiently small $\log \psi^{-1}(r) \asymp \log r^{-1}$ (see $\S 2.4$ ). Thus, within the iterated logarithms of $f_{\delta}(r)$, we could replace $\psi^{-1}(r)$ by $r^{-1}$ and thereby simplify the aesthetics of $f_{\delta}$.

The theorem shows that if condition (A) is satisfied then the sets $D(m, n ; \psi, \varphi)$ can be classified precisely via the dimension functions $f_{\delta}$ which are explicit in terms of $\psi^{-1}$ - the inverse of $\psi$ which exists since $\psi$ is strictly decreasing and continuous. Notice that if (A) is satisfied for some $k^{\prime}$, then it is also satisfied for any $k>k^{\prime}$ since $\psi$ is a decreasing function. However, the larger the $k$, the more intricate the dimension functions, and the stronger the condition becomes that $r^{m+n} \psi(r)^{n} \prod_{i=1}^{k-1} \log _{i} r$ is non-increasing. This latter condition is natural given our context - in the classical statements concerning the metric structure of the sets $W(m, n ; \psi)$ (see $\S 2.1)$ the condition that $r^{m+n} \psi(r)^{n}$ is non-increasing is standard and excludes the possibility of $W(m, n ; \psi)$ being the whole ambient space $\mathbb{R}^{m n}$. In these classical statements the dimension function is simply a power of $r$ so in our situation if we were to exclude the iterated logarithms in $f_{\delta}$ (which is the case when $\mathrm{k}=1$ ) then the two conditions naturally coincide.

We suspect that if condition (A) in Theorem 3 were to be replaced by any thing weaker, then given the generality, one would lose the delicate 'zero-infinity' law with respect to explicit dimension functions $f_{\delta}$. However, one should still be able to draw precise conclusions regarding the dimension of $D(m, n ; \psi, \varphi)$. We will return to this discussion later.

Example 1: The exact t-logarithmic order situation. With $\alpha_{o}>(m+n) / n$ and $t \geq 1$, consider the functions

$$
\psi(r)=r^{-\alpha_{o}} \prod_{i=1}^{t}\left(\log _{i} r\right)^{-\alpha_{i}} \quad \text { and } \varphi(r)=\psi(r) /\left(\log _{t} r\right)^{\alpha} \quad(\alpha>0) .
$$

So, for $r$ sufficiently small, we have that

$$
\frac{\varphi(r)}{\psi\left(r \log _{k} r\right)} \ll \frac{\left(\log _{k} r\right)^{\alpha_{o}}}{\left(\log _{t} r\right)^{\alpha}}<1 \quad \text { if } \quad k \geq t+1
$$


It is easy to check that $\lambda(\psi)=\alpha_{o}$ and that $\psi^{-1}(r) \asymp r^{-\frac{1}{\alpha_{o}}} \prod_{i=1}^{t}\left(\log _{i} r^{-1}\right)^{-\frac{\alpha_{i}}{\alpha_{o}}}$. Thus, with $k=t+1$ in condition (A), Theorem 3 implies the zero-infinity law with respect to the dimension function $f_{\delta}$ as defined in $\S 1.3$ and that $\operatorname{dim} D(m, n ; \psi, \varphi)=$ $n(m-1)+(m+n) / \alpha_{0}$.

Example 2: Functions not expressible as a product of iterated logarithms. With $\tau>(m+n) / n$ and $t \geq 1$, consider the functions

$$
\psi(r)=r^{-\tau}(\log r)^{\log \log r} \text { and } \varphi(r)=\psi(r) /\left(\log _{t} r\right)^{\alpha} \quad(\alpha>0) .
$$

So, for $r$ sufficiently small, we have that

$$
\frac{\varphi(r)}{\psi\left(r \log _{k} r\right)}=\frac{(\log r)^{\log \log r}}{\left(\log \left(r \log _{k} r\right)\right)^{\log \log \left(r \log _{k} r\right)}} \times \frac{\left(\log _{k} r\right)^{\tau}}{\left(\log _{t} r\right)^{\alpha}}<1 \quad \text { if } k \geq t+1 .
$$

It is easy to check that $\lambda(\psi)=\tau$ and that $\psi^{-1}(r) \asymp r^{-\frac{1}{\tau}}\left(\log r^{-1}\right)^{\frac{1}{\tau} \log \log r^{-1}}$. Thus, with $k=t+1$ in condition (A), Theorem 3 implies the zero-infinity law with respect to the dimension function $f_{\delta}$ given by

$$
f_{\delta}(r)=r^{n(m-1)+\frac{m+n}{\tau}}\left(\log r^{-1}\right)^{-\frac{m+n}{\tau} \log \log r^{-1}} \prod_{i=1}^{t-1}\left(\log _{i} r^{-1}\right)^{-1}\left(\log _{t} r^{-1}\right)^{-1-\delta},
$$

and that $\operatorname{dim} D(m, n ; \psi, \varphi)=n(m-1)+(m+n) / \tau$.

Example 3: Exponential functions. With $\tau>0$ and $t \geq 1$, let

$$
\psi(r)=\exp \left(-r^{\tau}\right) \text { and } \varphi(r)=\exp \left(-r^{\tau} \log _{t} r\right) .
$$

So, for $r$ sufficiently small, we have that

$$
\frac{\varphi(r)}{\psi\left(r \log _{k} r\right)}=\exp \left(-r^{\tau}\left(\log _{t} r-\left(\log _{k} r\right)^{\tau}\right)\right)<1 \quad \text { if } k \geq t+1
$$

It is easy to see that $\lambda(\psi)=\infty$ and that $\psi^{-1}(r)=\left(\log r^{-1}\right)^{\frac{1}{\tau}}$. Thus, with $k=t+1$ in condition (A), Theorem 3 implies the zero-infinity law with respect to the dimension function $f_{\delta}$ given by

$$
f_{\delta}(r)=r^{n(m-1)}\left(\log r^{-1}\right)^{-\frac{m+n}{\tau}} \prod_{i=2}^{t}\left(\log _{i} r^{-1}\right)^{-1}\left(\log _{t+1} r^{-1}\right)^{-1-\delta}
$$

and that $\operatorname{dim} D(m, n ; \psi, \varphi)=n(m-1)$. Notice, that if $\lambda(\psi)=\infty$ then the dimension of $D(m, n ; \psi, \varphi)$ is only dependent on $m$ and $n$. Thus, in such cases the discriminating information concerning the 'size' of the sets $D(m, n ; \psi, \varphi)$ is completely contained within the dimension functions $f_{\delta}$ via the zero-infinity laws.

Acknowledgments. SV would like to thank Martin Collins for doing all those 'other things' while this paper was coming together - thank you. He would also like to thank Duncan Bennett for reading through the final drafts. 
We would like to thank the referee for prompting us to 'find' the general formalism which appears as $\S 1.4$.

\section{$\S 2$ Proof of Theorems.}

We begin by stating two key results which are central to our approach.

\section{$\S 2.1$ Auxiliary Results.}

We require the following results concerning the 'size' of the set of $\psi$-well approximable points lying within the $m n$-dimensional unit cube $I^{m n}:=[0,1]^{m n}$. This 'restricted' set of $\psi$-well approximable points will be denoted by $\widetilde{W}(m, n ; \psi)$, thus $\widetilde{W}(m, n ; \psi):=W(m, n ; \psi) \cap I^{m n}$. The first of these results relates the size of $\widetilde{W}(m, n ; \psi)$, expressed in terms of $m n$-dimensional Lebesgue measure, to the behaviour of a particular 'volume' sum [5].

Theorem (Khintchine-Groshev). Let $\psi: \mathbb{R}^{+} \rightarrow \mathbb{R}^{+}$be a positive decreasing function and suppose that for $m=1$ and $m=2, \quad r^{m+n} \psi(r)^{n}$ is decreasing. Then

$$
|\widetilde{W}(m, n ; \psi)|_{m n}=\left\{\begin{array}{ccc}
0 & \text { if } \quad \sum \psi(r)^{n} & r^{m+n-1}<\infty \\
1 & \text { if } \quad \sum \psi(r)^{n} & r^{m+n-1}=\infty
\end{array}\right.
$$

The next result is a Hausdorff measure version of the above theorem [2].

Theorem (DV). Let $f$ be a dimension function such that $r^{-m n} f(r) \rightarrow \infty$ as $r \rightarrow 0$ and $r^{-m n} f(r)$ is non-increasing. Let $\psi: \mathbb{R}^{+} \rightarrow \mathbb{R}^{+}$be a decreasing function such that $r^{m+n} \psi(r)^{n} \rightarrow 0$ as $r \rightarrow \infty$ and $r^{m+n} \psi(r)^{n}$ is non-increasing. Furthermore, suppose that $r^{m+n} \psi(r)^{-(m-1) n} f(\psi(r))$ is non-increasing. Then

$$
\mathcal{H}^{f}(\widetilde{W}(m, n ; \psi))=\left\{\begin{array}{ccccc}
0 & \text { if } & \sum_{r=1}^{\infty} f(\psi(r)) & \psi(r)^{-(m-1) n} & r^{m+n-1}<\infty \\
\infty & \text { if } & \sum_{r=1}^{\infty} f(\psi(r)) & \psi(r)^{-(m-1) n} & r^{m+n-1}=\infty
\end{array} .\right.
$$

Notice, that the case when $\mathcal{H}^{f}$ is comparable to $m n$-dimensional Lebesgue measure (i.e. $f(r)=r^{m n}$ ) is excluded by the condition $r^{-m n} f(r) \rightarrow \infty$ as $r \rightarrow 0$. This will be significant in establishing Theorem 2. The condition $r^{m+n} \psi(r)^{n} \rightarrow 0$ as $r \rightarrow \infty$ is a natural one as if this condition was not obeyed then the set would have full Lebesgue measure by the Khintchine-Groshev theorem. The proofs of Theorem 1 and 3 make use of only the latter result, while Theorem 2 relies on both.

A comment on the convergence part. Most of the conditions on $f$ and $\psi$ in the above theorem are only required in establishing the divergence case. The convergence case follows from a straightforward application of the standard BorelCantelli lemma and for this the only necessary condition is that $\psi$ is decreasing and tending to zero (see [2]). This fact will be used in proving Theorem 3. 
Remark. It is easy to verify that $\psi$-approximability is not affected under translations by integer matrices and that the measures $\mathcal{H}^{f}$ are invariant under translation. Thus $\mathcal{H}^{f}(W(m, n ; \psi))=0$ whenever $\mathcal{H}^{f}(\widetilde{W}(m, n ; \psi))=0$ and $\mathcal{H}^{f}(W(m, n ; \psi))=$ $\infty$ whenever $\mathcal{H}^{f}(\widetilde{W}(m, n ; \psi))>0$. In particular, if $|\widetilde{W}(m, n ; \psi)|_{m n}=1$ then $|W(m, n ; \psi)|_{m n}=\infty$. Clearly, the same statements are valid for the sets $E_{t}\left(m, n ; \alpha_{t}\right)$ and $\widetilde{E}_{t}\left(m, n ; \alpha_{t}\right)$.

\section{$\S 2.2$ Proof of Theorem 1 .}

We begin by establishing the measure part of the statement for the dimension function $f_{\delta}$ with $\delta=0$. To simplify notation we will write $f$ for $f_{o}$ throughout.

For $\alpha_{o} \geq \frac{m+n}{n}$ and $\eta \geq 0$, consider the function:

$$
\psi_{\eta}(r)=r^{-\alpha_{o}} \prod_{i=1}^{t}\left(\log _{i} r\right)^{-\alpha_{i}} \times\left(\log _{t+1} r\right)^{-\frac{\alpha_{o}}{m+n}(1+\eta)} .
$$

We now calculate the $f$-dimension Hausdorff measure $\mathcal{H}^{f}$ of the set $W\left(m, n ; \psi_{\eta}\right)$ of $\psi_{\eta}$-well approximable points. In order to apply Theorem DV the expression

$$
f\left(\psi_{\eta}(r)\right)\left(\psi_{\eta}(r)\right)^{-(m-1) n} r^{m+n-1}
$$

must be considered.

If $t=0$ then $f(r)=r^{d}$ and

- $f\left(\psi_{\eta}(r)\right)=r^{-\alpha_{o} d}(\log r)^{-\frac{\alpha_{o} d}{m+n}(1+\eta)}$

- $\psi_{\eta}(r)^{-(m-1) n}=r^{\alpha_{o}(m-1) n}(\log r)^{\frac{\alpha_{o}(m-1) n}{m+n}(1+\eta)}$.

Thus for $t=0$, expression (2) is equal to $r^{-1}(\log r)^{-(1+\eta)}$. Now suppose that $t \geq 1$, so

$$
f(r):=r^{d} \prod_{i=1}^{t}\left(\log _{i} r^{-1}\right)^{u\left(\alpha_{i}\right)} .
$$

For $1 \leq j \leq t$ and $r$ sufficiently large

$$
\begin{aligned}
\log _{j} \psi_{\eta}(r)^{-1} & =\log _{j-1}\left(\alpha_{o} \log r+\sum_{i=1}^{t} \alpha_{i} \log _{i+1} r+\frac{\alpha_{o} d}{m+n}(1+\eta) \log _{t+2} r\right) \\
& \asymp \log _{j} r .
\end{aligned}
$$

Hence for $r$ sufficiently large

$$
f\left(\psi_{\eta}(r)\right) \asymp r^{-\alpha_{o} d} \prod_{i=1}^{t}\left(\log _{i} r\right)^{-\alpha_{i} d+u\left(\alpha_{i}\right)}\left(\log _{t+1} r\right)^{-\frac{\alpha_{o} d}{m+n}(1+\eta)},
$$

giving that expression (2) is comparable to

$$
r^{-1} \prod_{i=1}^{t}\left(\log _{i} r\right)^{-1}\left(\log _{t+1} r\right)^{-(1+\eta)} .
$$


Thus for $t \geq 0$, the sum in Theorem DV diverges if $\eta=0$ and converges for $\eta>0$. Now clearly

$$
\begin{aligned}
r^{-m n} f(r) & =r^{\frac{m+n}{\alpha_{o}}-n} \prod_{i=1}^{t}\left(\log _{i} r^{-1}\right)^{u\left(\alpha_{i}\right)}, \\
r^{m+n} & \psi_{\eta}(r)^{n}=r^{-\alpha_{o} n+m+n} \prod_{i=1}^{t}\left(\log _{i} r\right)^{-\alpha_{i} n} \times\left(\log _{t+1} r\right)^{-\frac{\alpha_{o} n}{m+n}(1+\eta)},
\end{aligned}
$$

and for $r$ sufficiently large

$$
f\left(\psi_{\eta}(r)\right)\left(\psi_{\eta}(r)\right)^{-(m-1) n} r^{m+n} \asymp \prod_{i=1}^{t}\left(\log _{i} r\right)^{-1}\left(\log _{t+1} r\right)^{-(1+\eta)} \rightarrow 0 \text { as } r \rightarrow \infty .
$$

When $t=0$, the above product terms for $i=1$ to $t$ are set to one. Here, we have stated the obvious but (3) and (4) are central to the conditions imposed in Theorem 2 and also clarify the following. Under the assumption that $\alpha_{o}>(m+n) / n$, it is easily seen that the functions $f$ and $\psi_{\eta}$ satisfy the conditions of Theorem DV. Thus

$$
\mathcal{H}^{f}\left(W\left(m, n ; \psi_{o}\right)\right)=\infty \quad \text { and } \quad \mathcal{H}^{f}\left(W\left(m, n ; \psi_{\eta>0}\right)\right)=0 .
$$

Now, for $\eta>0$ consider the set

$$
D_{t}\left(m, n ; \psi_{\eta}\right):=W\left(m, n ; \psi_{o}\right) \backslash W\left(m, n ; \psi_{\eta}\right) .
$$

The above argument implies that

$$
\mathcal{H}^{f}\left(D_{t}\left(m, n ; \psi_{\eta}\right)\right)=\infty .
$$

Suppose $X \in D_{t}\left(m, n ; \psi_{\eta}\right)$, then

$$
|\mathbf{q} X-\mathbf{p}| \leq|\mathbf{q}|^{-\alpha_{o}+1} \prod_{i=1}^{t}\left(\log _{i}|\mathbf{q}|\right)^{-\alpha_{i}} \times\left(\log _{t+1}|\mathbf{q}|\right)^{-\frac{\alpha_{o}}{m+n}}
$$

for infinitely many $(\mathbf{p}, \mathbf{q}) \in \mathbb{Z}^{n} \times \mathbb{Z}^{m}$, and

$$
|\mathbf{q} X-\mathbf{p}| \geq|\mathbf{q}|^{-\alpha_{o}+1} \prod_{i=1}^{t}\left(\log _{i}|\mathbf{q}|\right)^{-\alpha_{i}} \times\left(\log _{t+1}|\mathbf{q}|\right)^{-\frac{\alpha_{o}}{m+n}(1+\eta)}
$$

for all $(\mathbf{p}, \mathbf{q}) \in \mathbb{Z}^{n} \times \mathbb{Z}^{m}$ with $|\mathbf{q}|$ sufficiently large. It follows from the definition of $E_{t}\left(m, n ; \alpha_{t}\right)$, that $X \in E_{t}\left(m, n ; \alpha_{t}\right)$ and so $D_{t}\left(m, n ; \psi_{\eta}\right) \subseteq E_{t}\left(m, n ; \alpha_{t}\right)$. Thus, in view of (5) we obtain that

$$
\mathcal{H}^{f}\left(E_{t}\left(m, n ; \alpha_{t}\right)\right)=\infty
$$

The dimension part of the theorem will now be established. For any $\epsilon>0$ and $r$ sufficiently small $f(r) \leq r^{d-\epsilon}$. Hence

$$
\mathcal{H}^{d-\epsilon}\left(E_{t}\left(m, n ; \alpha_{t}\right)\right) \geq \underset{11}{\mathcal{H}^{f}}\left(E_{t}\left(m, n ; \alpha_{t}\right)\right)=\infty
$$


which implies that $\operatorname{dim} E_{t}\left(m, n ; \alpha_{t}\right) \geq d-\epsilon$. However, $\epsilon>0$ is arbitrary and so on letting $\epsilon \rightarrow 0$ the lower bound

$$
\operatorname{dim} E_{t}\left(m, n ; \alpha_{t}\right) \geq d
$$

is obtained. For the upper bound, we first note that for any $\epsilon>0$,

$$
E_{t}\left(m, n ; \alpha_{t}\right) \subseteq W\left(m, n ; \alpha_{o}-\epsilon\right) .
$$

Now on applying Theorem DV, it is easy to verify that

$$
\operatorname{dim} E_{t}\left(m, n ; \alpha_{t}\right) \leq \operatorname{dim} W\left(m, n ; \alpha_{o}-\epsilon\right)=(m-1) n+\frac{m+n}{\alpha_{o}-\epsilon} ;
$$

essentially, Theorem DV implies that $\operatorname{dim} W(m, n ; \psi)$ is the exponent of convergence of $\sum \psi(r)^{s-(m-1) n} r^{m+n-1}-$ see [2] if necessary. Thus, on letting $\epsilon \rightarrow 0$ we obtain the upper bound $\operatorname{dim} E_{t}\left(m, n ; \alpha_{t}\right) \leq d$. This together with (6) completes the proof of the dimension part.

To complete the proof of the theorem we must show that $\mathcal{H}^{f_{\delta}}\left(E_{t}\left(m, n ; \alpha_{t}\right)\right)=0$ for dimension functions $f_{\delta}$ with $\delta>0$. When $t=0, f_{\delta}(r):=r^{d+\delta}$ and the required statement follows trivially from the definition of Hausdorff dimension. Without loss of generality assume that $t \geq 1$. For $\epsilon>0$, consider the function $\psi_{\epsilon}$ given by

$$
\psi_{\epsilon}(r):=r^{-\alpha_{o}} \prod_{i=1}^{t}\left(\log _{i} r\right)^{-\alpha_{i}} \times\left(\log _{t} r\right)^{\epsilon} .
$$

It follows, by definition, that $E_{t}\left(m, n ; \alpha_{t}\right) \subseteq W\left(m, n ; \psi_{\epsilon}\right)$ and so

$$
\mathcal{H}^{f_{\delta}}\left(E_{t}\left(m, n ; \alpha_{t}\right)\right) \leq \mathcal{H}^{f_{\delta}}\left(W\left(m, n ; \psi_{\epsilon}\right)\right) .
$$

We now show that $\mathcal{H}^{f_{\delta}}\left(W\left(m, n ; \psi_{\epsilon}\right)\right)=0$ which together with the above statement implies the desired result. In terms of the function $f:=f_{o}$, we have that $f_{\delta}(r)=$ $f(r)\left(\log _{t} r^{-1}\right)^{-\delta}$. It can be verified that for $r$ sufficiently large

$$
f_{\delta}\left(\psi_{\epsilon}(r)\right) \asymp r^{-\alpha_{o} d} \prod_{i=1}^{t}\left(\log _{i} r\right)^{-\alpha_{i} d+u\left(\alpha_{i}\right)}\left(\log _{t} r\right)^{-\delta+\epsilon d},
$$

and so

$$
f_{\delta}\left(\psi_{\epsilon}(r)\right)\left(\psi_{\epsilon}(r)\right)^{-(m-1) n} r^{m+n-1} \asymp r^{-1} \prod_{i=1}^{t}\left(\log _{i} r\right)^{-1} \times\left(\log _{t} r\right)^{\frac{(m+n) \epsilon}{\alpha_{o}}-\delta} .
$$

Now assume that $0<\epsilon<\frac{\delta \alpha_{o}}{m+n}$. Since $\alpha_{o}>(m+n) / n$, the functions $f_{\delta}$ and $\psi_{\epsilon}$ satisfy the conditions of Theorem DV and with $\epsilon$ as above we have that $\sum f_{\delta}\left(\psi_{\epsilon}(r)\right)\left(\psi_{\epsilon}(r)\right)^{-(m-1) n} r^{m+n-1}<\infty$. Hence, Theorem DV implies that

$$
\mathcal{H}^{f_{\delta}}\left(W\left(m, n ; \psi_{\epsilon}\right)\right)=0
$$

and completes the proof of Theorem 1 . 


\section{$\S 2.3$ Proof of Theorem 2 .}

Since $\alpha_{o}=(m+n) / n$, we have that $u\left(\alpha_{i}\right)=\alpha_{i} n-1$ and $d=m n$.

First, suppose that $t=0$ or $\alpha_{i}=1 / n$ for all $1 \leq i \leq t$. Then $f_{o}(r)=r^{m n}$ and the measure $\mathcal{H}^{f_{o}}$ is comparable to $m n$-dimensional Lebesgue measure. Let $\psi_{\eta}$ be given by (1) and notice that

$$
r^{m+n} \psi_{\eta}(r)^{n}=\prod_{i=1}^{t}\left(\log _{i} r\right)^{-1} \times\left(\log _{t+1} r\right)^{-(1+\eta)} \rightarrow 0 \quad \text { as } \quad r \rightarrow \infty .
$$

Hence, the Khintchine--Groshev Theorem implies that

$$
\left|\widetilde{W}\left(m, n ; \psi_{\eta}\right)\right|_{m n}=\left\{\begin{array}{ccc}
0 & \text { if } & \eta>0 \\
1 & \text { if } & \eta=0
\end{array} .\right.
$$

On following the same line of arguments as in the proof of Theorem 1, we find that the above statement implies that

$$
\left|\widetilde{E}_{t}\left(m, n ; \alpha_{t}\right)\right|_{m n}=1 .
$$

This in turn, implies that $\left|E_{t}\left(m, n ; \alpha_{t}\right)\right|_{m n}=\infty$ (if necessary, see remark at end of $\S 2.1)$ and that $\operatorname{dim} E_{t}\left(m, n ; \alpha_{t}\right) \geq m n$. Equality for the dimension now follows trivially since $E_{t}\left(m, n ; \alpha_{t}\right) \subseteq \mathbb{R}^{m n}$. Next, let $h$ be any dimension function such that $r^{-m n} h(r) \rightarrow 0$ as $r \rightarrow 0$. Then (8) together with the fact in $\S 1.3$, implies that $\mathcal{H}^{h}\left(\widetilde{E}_{t}\left(m, n ; \alpha_{t}\right)\right)=0$. This completes the proof of the theorem in the case $t=0$ or $\alpha_{i}=1 / n$ for all $1 \leq i \leq t$. We now show that in all other cases $\left|E_{t}\left(m, n ; \alpha_{t}\right)\right|_{m n}=$ 0 .

With $t \geq 1$, let $k$ be the unique integer in $[1, t]$ such that $\alpha_{k} \neq 1 / n$ and $\alpha_{i}=1 / n$ for all $1 \leq i \leq k-1$. If $\alpha_{k}>1 / n$, the result that $\left|E_{t}\left(m, n ; \alpha_{t}\right)\right|_{m n}=0$ follows directly from the last part of the theorem (proved below). More precisely, for $\delta>0$, since $r^{m n} / f_{\delta}(r) \rightarrow 0$ as $r \rightarrow 0$ (one needs that $\delta<\alpha_{t} n-1$ if $k=t$ ) and $\mathcal{H}^{f_{\delta}}\left(E_{t}\left(m, n ; \alpha_{t}\right)\right)=0$ the fact in $\S 1.3$ implies the desired conclusion. So, assume without loss of generality that $\alpha_{k}<1 / n$ and for $0<\epsilon<1 / n-\alpha_{k}$ set

$$
\psi_{ \pm \epsilon}(r)=r^{-\alpha_{o}} \prod_{i=1}^{t}\left(\log _{i} r\right)^{-\alpha_{i}} \times\left(\log _{t} r\right)^{ \pm \epsilon}
$$

Note that $\psi_{\epsilon}$ is the standard function given in (7). By definition $\widetilde{E}_{t}\left(m, n ; \alpha_{t}\right) \subset$ $\widetilde{W}\left(m, n ; \psi_{\epsilon}\right) \backslash \widetilde{W}\left(m, n ; \psi_{-\epsilon}\right)$. Suppose for the moment that

$$
r^{m+n} \psi_{-\epsilon}(r)^{n}=\prod_{i=1}^{t}\left(\log _{i} r\right)^{-\alpha_{i} n} \times\left(\log _{t} r\right)^{-\epsilon n} \rightarrow 0 \quad \text { as } \quad r \rightarrow \infty,
$$

then the Khintchine-Groshev Theorem implies that $\left|\widetilde{W}\left(m, n ; \psi_{-\epsilon}\right)\right|_{m n}=1$. If $r^{m+n} \psi_{-\epsilon}(r)^{n} \not 0$ as $r \rightarrow \infty$ (for example if $k=1$ and $\left.\alpha_{1}<0\right)$ then $\widetilde{W}\left(m, n ; \alpha_{o}\right) \subseteq$ $\widetilde{W}\left(m, n ; \psi_{-\epsilon}\right)$ and Dirichlet's Theorem for linear forms implies that $\widetilde{W}\left(m, n ; \psi_{-\epsilon}\right)=$ 
$I^{m n}$. The upshot of this is that $\left|\widetilde{W}\left(m, n ; \psi_{-\epsilon}\right)\right|_{m n}=1$ always. Since $\widetilde{W}\left(m, n ; \psi_{-\epsilon}\right) \subseteq$ $\widetilde{W}\left(m, n ; \psi_{\epsilon}\right)$ we also have that $\left|\widetilde{W}\left(m, n ; \psi_{\epsilon}\right)\right|_{m n}=1$ and so $\left|\widetilde{E}_{t}\left(m, n ; \alpha_{t}\right)\right|_{m n}=0$ as claimed.

To complete the proof of theorem we need to establish the 'in particular' part. So let $k$ be defined as above with $\alpha_{k}>1 / n$. Then,

$$
r^{-m n} f_{o}(r)=\prod_{i=k}^{t}\left(\log _{i} r^{-1}\right)^{u\left(\alpha_{i}\right)} \rightarrow \infty \text { as } r \rightarrow 0,
$$

and so with $\psi_{\eta}$ given by (1) the conditions of Theorem DV are satisfied. On following exactly the same line of arguments as in the proof of Theorem 1 we deduce that $\mathcal{H}^{f_{o}}\left(E_{t}\left(m, n ; \alpha_{t}\right)\right)=\infty$ and that $\operatorname{dim} E_{t}\left(m, n ; \alpha_{t}\right) \geq m n$. Of course, the upper bound for the dimension is trivial since $E_{t}\left(m, n ; \alpha_{t}\right) \subseteq \mathbb{R}^{m n}$.

We now show that $\mathcal{H}^{f_{\delta}}\left(E_{t}\left(m, n ; \alpha_{t}\right)\right)=0$ for the dimension function $f_{\delta}$ with $\delta>0$. Let $\psi_{\epsilon}$ be given by $(7)$, so by definition $E_{t}\left(m, n ; \alpha_{t}\right) \subseteq W\left(m, n ; \psi_{\epsilon}\right)$ and hence it is only necessary to show that $\mathcal{H}^{f_{\delta}}\left(W\left(m, n ; \psi_{\epsilon}\right)\right)=0$. The proof is essentially the same as in Theorem 1 with a slight twist.

Fix $\delta>0$ and choose $\epsilon<\delta / n$. Assume for the moment that either $k<t$ or $k=t$ and $\delta<n \alpha_{t}-1$. It can then be verified that the conditions of Theorem DV are satisfied for the functions $f_{\delta}$ and $\psi_{\epsilon}$. In particular the sum in Theorem DV converges and so $\mathcal{H}^{f_{\delta}}\left(W\left(m, n ; \psi_{\epsilon}\right)\right)=0$ as required. Now suppose that $k=t$ and $\delta>n \alpha_{t}-1$. In this case $r^{-m n} f_{\delta}(r) \rightarrow 0$ as $r \rightarrow 0$ and so Theorem DV cannot be applied. However, with $0<\epsilon<\alpha_{t}-1 / n$ the conditions of the Khintchine-Groshev Theorem are satisfied and as a consequence we obtain that $\left|W\left(m, n ; \psi_{\epsilon}\right)\right|_{m n}=0$ since the sum converges. This together with the fact from $\S 1.3$ implies that $\mathcal{H}^{f_{\delta}}\left(W\left(m, n ; \psi_{\epsilon}\right)\right)=0$ as required.

The proof of Theorem 2 is now complete.

\section{$\S 2.4$ Proof of Theorem 3.}

We will prove the theorem under the assumption that $k \geq 2$ in condition (A). The case when $k=1$ follows with the obvious modifications and no new ideas are required.

We begin by studying the function $f_{\delta}$ stated in the theorem; in particular the behaviour of $f_{\delta}(r)$ as $r$ tends to zero since this together with the measure part of the theorem implies the dimension part. The fact that the function $f_{\delta}$ given by

$$
f_{\delta}(r):=r^{(m-1) n}\left(\psi^{-1}(r)\right)^{-m-n} \prod_{i=1}^{k-2}\left(\log _{i} \psi^{-1}(r)\right)^{-1} \times\left(\log _{k-1} \psi^{-1}(r)\right)^{-1-\delta}
$$

is a dimension function is easily seen. As $\psi(r)$ is strictly decreasing, continuous and tending to zero as $r \rightarrow \infty$ (since the order $\lambda(\psi)>0$ ) we have that the inverse $\psi^{-1}(r)$ is also strictly decreasing, continuous and tending to infinity as $r \rightarrow 0$. Thus 
$\left(\psi^{-1}(r)\right)^{-m-n}$ and $\prod_{i=1}^{k-2}\left(\log _{i} \psi^{-1}(r)\right)^{-1}\left(\log _{k-1} \psi^{-1}(r)\right)^{-1-\delta}$ are all continuous, increasing with $r$ and tend to zero as $r \rightarrow 0$. Thus, the function $f_{\delta}$ is continuous, increasing with $r$ and $f_{\delta}(r) \rightarrow 0$ as $r \rightarrow 0$; i.e. $f_{\delta}$ is a dimension function. By the definition of $\lambda(\psi)$, it follows that

$$
\lim _{r \rightarrow 0} \frac{\log \left(\psi^{-1}(r)\right)}{-\log r}=\frac{1}{\lambda(\psi)} .
$$

This in turn implies that for the function $f_{\delta}(\delta \geq 0)$

$$
\lim _{r \rightarrow 0} \frac{\log f_{\delta}(r)}{\log r}=d(\psi):=n(m-1)+(m+n) / \lambda(\psi) ;
$$

i.e. given any $\epsilon>0$ we have that for $r$ sufficiently small

$$
r^{d(\psi)+\epsilon}<f_{\delta}(r)<r^{d(\psi)-\epsilon} .
$$

On assuming the measure part of the theorem, we conclude that for any $\epsilon>0$

$$
\begin{gathered}
\infty=\mathcal{H}^{f_{o}}(D(m, n ; \psi, \varphi)) \leq \mathcal{H}^{d(\psi)-\epsilon}(D(m, n ; \psi, \varphi)) \\
\Longrightarrow \operatorname{dim} D(m, n ; \psi, \varphi) \geq d(\psi)-\epsilon ;
\end{gathered}
$$

and if $\delta>0$ then

$$
\begin{gathered}
0=\mathcal{H}^{f_{\delta}}(D(m, n ; \psi, \varphi)) \geq \mathcal{H}^{d(\psi)+\epsilon}(D(m, n ; \psi, \varphi)) \\
\Longrightarrow \operatorname{dim} D(m, n ; \psi, \varphi) \leq d(\psi)+\epsilon .
\end{gathered}
$$

The dimension part of the theorem now follows on letting $\epsilon \rightarrow 0$ in the above inequalities for $\operatorname{dim} D(m, n ; \psi, \varphi)$. Alternatively, as in the proof of Theorem 1 , the upper bound for dimension can also be easily deduced from the fact that $D(m, n ; \psi, \varphi) \subseteq W(m, n ; \lambda(\psi)-\epsilon)$.

We now establish the measure part of the theorem, i.e. the zero-infinity law with respect to the dimension functions $f_{\delta}$. The strategy is simple enough and to some extent follows the proof of Theorem 1 . The basic idea is to show that

$$
\mathcal{H}^{f_{\delta}}(W(m, n ; \psi))=\infty \text { and } \quad \mathcal{H}^{f_{\delta}}(W(m, n ; \varphi))=0 \quad \text { if } \quad \delta=0,
$$

and that

$$
\mathcal{H}^{f_{\delta}}(W(m, n ; \psi))=0 \quad \text { if } \quad \delta>0 .
$$

The stated zero-infinity law then follows from the definition of $D(m, n ; \psi, \varphi)$ and the trivial fact that $D(m, n ; \psi, \varphi) \subseteq W(m, n ; \psi)$.

We begin by considering the sum which appears in Theorem DV. As $f_{\delta}(\psi(r))=$ $r^{-(m+n)} \psi(r)^{(m-1) n} \prod_{i=1}^{k-2}\left(\log _{i} r\right)^{-1}\left(\log _{k-1} r\right)^{-(1+\delta)}$ it follows that

$$
\sum_{r=1}^{\infty} f_{\delta}(\psi(r))(\psi(r))^{-(m-1) n} r^{m+n-1}\left\{\begin{array}{ll}
=\infty & \text { if } \delta=0 \\
<\infty & \text { if } \delta>0
\end{array} .\right.
$$


On the other hand, condition (A) implies that $\psi^{-1}(\varphi(r)) \geq r \log _{k} r$. Hence

$$
\begin{aligned}
& \sum_{r=1}^{\infty} f_{\delta}(\varphi(r))(\varphi(r))^{-(m-1) n} r^{m+n-1} \\
& \quad \leq \sum_{r=1}^{\infty} r^{m+n-1}\left(\psi ^ { - 1 } ( \varphi ( r ) ) ^ { - ( m + n ) } \prod _ { i = 1 } ^ { k - 1 } \left(\log _{i} \psi^{-1}(\varphi(r))^{-1}\right.\right. \\
& \quad \ll \sum_{r=1}^{\infty} r^{-1} \prod_{i=1}^{k-1}\left(\log _{i} r\right)^{-1}\left(\log _{k} r\right)^{-(m+n)}<\infty \quad \text { since } m+n \geq 2 .
\end{aligned}
$$

Now, both the functions $\psi$ and $\varphi$ are decreasing and tending to zero, thus the convergence part of Theorem DV implies that $\mathcal{H}^{f_{\delta}}(W(m, n ; \varphi))=0$ for $\delta \geq 0$ and $\mathcal{H}^{f_{\delta}}(W(m, n ; \psi))=0$ for $\delta>0$ - see comment on the convergence part of Theorem DV $(\S 2.1)$. It remains to show $\mathcal{H}^{f_{\delta}}(W(m, n ; \psi))=\infty$ when $\delta=0$. To simplify notation write $f$ for $f_{o}$. In view of (10), the desired statement will follow from the divergent part of Theorem DV provided that $f$ and $\psi$ satisfy the various conditions of Theorem DV. These we now verify.

The conditions that $r^{m+n} \psi(r)^{n} \rightarrow 0$ as $r \rightarrow \infty$ and $r^{m+n} \psi(r)^{n}$ is non-increasing follow respectively from the fact that the order $\lambda(\psi)>(m+n) / n$ and our hypothesis that

$$
g(r):=r^{m+n} \psi(r)^{n} \prod_{i=1}^{k-1} \log _{i} r
$$

is non-increasing. Next,

$$
r^{m+n} \psi(r)^{-(m-1) n} f(\psi(r))=\prod_{i=1}^{k-1}\left(\log _{i} r\right)^{-1}
$$

which is certainly non-increasing. This leaves verifying the conditions on $f$, namely that $r^{-m n} f(r) \rightarrow \infty$ as $r \rightarrow 0$ and $r^{-m n} f(r)$ is non-increasing as $r$ increases. It is easy to see that

$$
r^{-m n} f(r)=r^{-n}\left(\psi^{-1}(r)\right)^{-(m+n)} \prod_{i=1}^{k-1}\left(\log _{i} \psi^{-1}(r)\right)^{-1}=\left(g\left(\psi^{-1}(r)\right)\right)^{-1} .
$$

The fact that this is non-increasing follows from our hypothesis that $g(r)$ is nonincreasing. Briefly, $(g(r))^{-1}$ is non-decreasing and hence $\left(g\left(\psi^{-1}(r)\right)\right)^{-1}$ is nonincreasing, since $\psi^{-1}(r)$ is decreasing. It is worth mentioning that it is only in establishing this condition that we need to impose as a hypothesis that $g(r)$ is nonincreasing rather than the standard hypothesis that $r^{m+n} \psi(r)^{n}$ is non-increasing. All that now remains to prove the zero-infinity law is to verify the condition that

$$
r^{-m n} f(r) \rightarrow \infty \text { as } r \rightarrow 0
$$

Since $\lambda(\psi)>(m+n) / n$, we have that $n-(m+n) / \lambda(\psi)>0$. Choose $0<\epsilon<$ $n-(m+n) / \lambda(\psi)$. Then by $(9)$, it follows that

$$
r^{-m n} f(r) \geq r^{-n+\frac{m+n}{\lambda(\psi)}+\epsilon} \rightarrow \infty \text { as } r \rightarrow 0 .
$$


This completes the verification of the conditions of Theorem DV and therefore the proof of Theorem 3 .

\section{$\S 2.5$ Concluding remarks.}

A natural weakening of condition (A) in Theorem 3 is to replace it by the condition:

$$
\lim _{r \rightarrow \infty} \frac{1}{r} \psi^{-1}(\varphi(r))=\infty \quad ;
$$

i.e. for any constant $C>1, \exists r_{o}=r_{o}(C)$ such that $\varphi(r) \leq \psi(C r) \quad \forall r>r_{o}$. Obviously, condition (A) implies condition (B) and so (B) is less restrictive than (A). For example, condition (B) allows us to consider step functions. We suspect that in such generality, it is not possible to establish the delicate 'zero-infinity' law with respect to explicit dimension functions as in Theorem 3. However, using the same strategy as in the proof of Theorem 3, it should still be possible to prove the following claim.

Claim: Let $\psi$ be strictly decreasing and continuous with $\lambda(\psi)>(m+n) / n$ and such that $r^{m+n} \psi(r)^{n}$ is non-increasing. Let $\varphi$ be decreasing such that $\psi / \varphi$ is strictly increasing and that condition (B) is satisfied. Then

$$
\operatorname{dim} D(m, n ; \psi, \varphi)=n(m-1)+(m+n) / \lambda(\psi) .
$$

Of course the upper bound is obvious since $D(m, n ; \psi, \varphi) \subseteq W(m, n ; \lambda(\psi)-\epsilon)$. The lower bound would follow if we could prove the existence of a dimension function $f$ such that $f$ and $\psi$ satisfy the conditions of Theorem DV and

$$
\sum_{r=1}^{\infty} f(\theta(r))(\theta(r))^{-(m-1) n} r^{m+n-1}\left\{\begin{array}{ll}
=\infty & \text { if } \theta=\psi \\
<\infty & \text { if } \theta=\varphi
\end{array} .\right.
$$

It is not too difficult to prove the existence of dimension function $f$ so that the above condition on the sum is satisfied. However, there are various technical problems in establishing the two monotonicity conditions in Theorem DV. We hope to overcome these problems in the near future.

For the sake of simplicity we restrict the following discussion to the case when $m=n=1$ and we simply write $D(\psi, \varphi)$ for $D(m, n ; \psi, \varphi)$. We have shown that under reasonably mild growth conditions on the functions $\psi$ and $\varphi$ and the degree to which $\varphi$ is smaller than $\psi$, then $\operatorname{dim} D(\psi, \varphi)=\operatorname{dim} W(m, n ; \psi)=n(m-1)+$ $(m+n) / \lambda(\psi)$. However, the techniques developed in this paper completely fail if we let $\varphi=c \psi$ where $0<c<1$ is a constant. Essentially, as a minimum, we have to assume that $\psi(r) / \varphi(r) \rightarrow \infty$ as $r \rightarrow \infty$ for our methods to succeed in giving a lower bound for the dimension. The sets $D(\psi, c \psi)$ clearly have intrinsic interest. Yet, in order to discuss their full relevance it is necessary to introduce the set $\operatorname{Bad}(\psi)$. Define $\operatorname{Bad}(\psi)$ to be the set of $x \in \mathbb{R}$ such that

$$
|x-p / q| \leq \psi(q) \quad \text { infinitely often }
$$


and that

$$
|x-p / q| \geq c(x) \psi(q) \quad \text { for all rationals } p / q,
$$

where $c(x)>0$ is a constant dependent only on $x$. It is natural to think of $\operatorname{Bad}(\psi)$ as the set of 'badly approximable numbers' with respect to the function $\psi$. To clarify the use of this language consider the set $\operatorname{Bad}(\psi)$ with $\psi(r)=r^{-2}$. Then $\operatorname{Bad}(\psi)$ consists of real numbers $x$ such that $|x-p / q| \leq q^{-2}$ infinitely often; and $|x-p / q| \geq c(x) q^{-2}$ for all rationals $p / q$. By Dirichlet's Theorem the infinitely often statement is satisfied by all real numbers, so the set under consideration is precisely the classical set of badly approximable numbers. It is well known that this set is of zero Lebesgue measure but of Hausdorff dimension one. Now, the sets $D(\psi, c \psi)$ are rather interesting since $D(\psi, c \psi) \subset \operatorname{Bad}(\psi)$ and $D(\psi, c \psi) \rightarrow \operatorname{Bad}(\psi)$ as $c \rightarrow 0$. Thus, lower bounds for $\operatorname{dim} D(\psi, c \psi)$ are also lower bounds for $\operatorname{dim} \operatorname{Bad}(\psi)$ and moreover

$$
\operatorname{dim} D(\psi, c \psi) \rightarrow \operatorname{dim} \operatorname{Bad}(\psi)
$$

as $c \rightarrow 0$. As far as we are aware, no metric results are known for the general sets $\operatorname{Bad}(\psi)$. As a first step, it would be interesting to determine $\operatorname{dim} D(\psi, c \psi)$, and so $\operatorname{dim} \operatorname{Bad}(\psi)$, in the case $\psi(r)=r^{-\alpha}$ with $\alpha>2$. As mentioned earlier, new ideas and methods would have to be developed.

\section{References}

[1] A. Baker and W. M. Schmidt. Diophantine approximation and Hausdorff dimension. Proc. Lond. Math. Soc., (3) 21 (1970) 1-11.

[2] H. Dickinson and S. L. Velani. Hausdorff measure and linear forms. J. reine angew. Math., 490 (1997) 1-36.

[3] K. Falconer : Fractal Geometry: Mathematical Foundations and Applications. John Wiley \& Sons, (1990).

[4] R. Güting. On Mahler's function $\theta_{1}$. Michigan Math. J., 10 (1963), 161-179.

[5] V.G.Sprindžuk: Metric theory of Diophantine approximation (translated by R. A. Silverman), V. H. Winston \& Sons, Washington D.C. (1979).

Victor V. Beresnevich: Institute of Mathematics, Academy of Sciences of Belarus, 220072, Surganova 11, Minsk, Belarus.

Detta Dickinson: Department of Mathematics, University of York, Heslington, York Y01 5DD, England.

Sanju L. Velani: Department of Mathematics, Queen Mary, University of London, Mile End Road, London E1 4NS, England. 